\title{
Human Body Sensing: a Pervasive Approach by Implanted RFID Tags
}

\author{
Cecilia Occhiuzzi \\ DISP, University of Roma Tor Vergata \\ Via del Politecnico 1, 00133 Roma \\ Email:occhiuzzi@disp.uniroma2.it
}

\author{
Gaetano Marrocco \\ DISP, University of Roma Tor Vergata \\ Via del Politecnico 1, 00133 Roma \\ Email:marrocco@disp.uniroma2.it
}

\begin{abstract}
Starting from the physical evidence that passive RFID systems may be used as self-sensing devices, the feasibility of human monitoring by means of implanted tags is here investigated. The key parameters to master with the purpose to enhance the sensitivity of the implanted radiosensor have been theoretically analyzed and numerically applied to a specific medical problem, i.e. the continuous monitoring of liver cancer evolution. Preliminary experimental results with simplified phantoms corroborate the analysis.
\end{abstract}

\section{INTRODUCTION}

Beside the common logistic usages of the Radio Frequency Identification (RFID) technology, one of the most innovative and promising applications is the possibility to process the backscattering signals with the purpose to detect additional information about the target, such as its state and its evolution, without any specific embedded sensor or local power supply. The rationale of this idea, the sensor-less sensing approach, lies in the clear dependence of the tag's input impedance and of the radar cross section on the physical and geometrical features of the real target. When the object where the tag is attached on undergoes some changes along with the time, the tag's electrical features also change and these variations can be remotely detected by the reader [1].

The possibility to remotely monitor processes in evolution opens interesting opportunity in telemedicine and human health monitoring in general, especially concerning implantable devices. One or more battery-less RFID radiosensors could be implanted inside the human body in close proximity, for example, to a surgery-treated region and interrogated by an external reader. Based on the sensors response at different times (days or even hours) a map of the geometrical or chemical changes of the tissues could be produced, thus evaluating the healing process and possible complications, e.g. abnormal cell proliferations, edema and inflammatory events (Fig. 1). The idea to implant RFID passive tag into the human body is not new [2], but up to date it has been used only for identification purposes without attempting to achieve sensing.

Starting from the physical evidence for which different biological processes involve modification of tissues dielectric properties [3], the aim of this study is to discuss the feasibility of the system especially in term of sensing capabilities and communication link. Particular attention is here devoted to understand the key parameters to enhance and amplify the sensitivity and consequently to provide a first guideline for the design of body-implanted RFID sensor tags.

A possible application of the proposed sensing platform is the monitoring of liver cancer evolution. Due to the increased angiogenesis and disruption of vascular architecture, significant differences exist in fact between the dielectric properties of normal and malignant liver tissue [4].

Numerical simulations (FDTD modeling) and an early experimental validation corroborate the sensing approach and the reasonableness of the proposed platform.

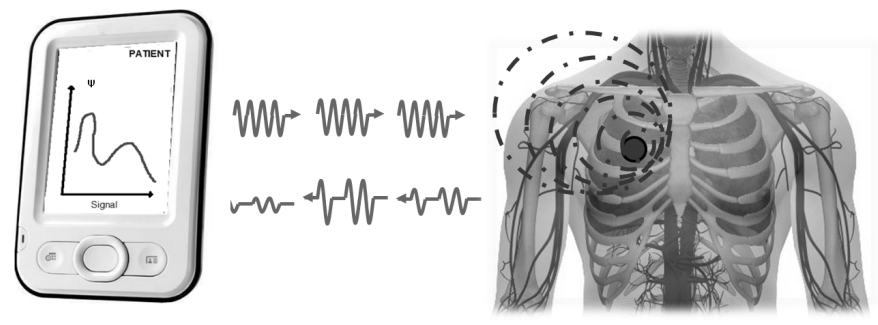

Fig. 1. A possible set-up for the Human Health Monitoring by implanted RFID technology.

\section{RFID-SENSING EQUATIONS}

We generally denote with $\Psi(t)$ a local physical or geometrical parameter of the body region which has to be monitored by the RFID platform. For the specific application $\Psi$ will be a shape factor of the biological process or the local effective permittivity.

The two-way reader-tag link may be hence rewritten making explicit the dependence on the variation of local parameters. The power collected at the microchip (1) and the power backscattered by the tag toward the reader (2) and collected by this are:

$$
\begin{gathered}
P_{R \rightarrow T}[\Psi]=\left(\frac{\lambda_{0}}{4 \pi d}\right)^{2} P_{i n} G_{R}(\theta, \phi) G_{T}[\theta, \phi, \Psi(t)] \tau[\Psi(t)] \eta_{p} \\
P_{R \leftarrow T}[\Psi]=\frac{1}{4 \pi}\left(\frac{\lambda_{0}}{4 \pi d}\right)^{2} P_{i n} G_{R}(\theta, \phi) \eta_{p}^{2} r \operatorname{rss}_{T}[\theta, \phi, \Psi(t)]
\end{gathered}
$$


where $d$ is the reader-tag distance, $G_{R}(\theta, \phi)$ is the gain of the reader antenna, $G_{T}[\theta, \phi, \Psi(t)]$ is the gain of the tag's antenna when placed onto the body. $P_{i n}$ is the power entering in the reader antenna, $\eta_{p}$ is the polarization mismatch between the reader and the tag, $\tau[\Psi(t)]$ is the power transmission coefficient of the tag:

$$
\tau[\Psi]=\frac{4 R_{\text {chip }} R_{A}[\Psi(t)]}{\left|Z_{\text {chip }}+Z_{A}[\Psi(t)]\right|^{2}}
$$

$r c s_{T}$ is the tag's radar cross-section, related to the modulation impedance $Z_{\text {mod }}$ of the microchip to encode the low and high digital state:

$$
\operatorname{rcs}_{T}[\Psi]=\frac{\lambda_{0}^{2}}{4 \pi} G_{T}^{2}[\theta, \phi, \Psi(t)]\left(\frac{2 R_{A}[\Psi(t)]}{\left|Z_{\text {mod }}+Z_{A}[\Psi(t)]\right|}\right)^{2}
$$

The backscattered power $P_{R \leftarrow T}$, directly measurable by the reader, is therefore strictly correlated to the physical variation of the local environment through the change of impedance and gain of the tag. In particular, when the reader-tag mutual position and $P_{i n}$ remain the same in successive interrogations, it is possible to simply derive a data inversion curve $p_{s} \leftrightarrow \Psi(t)$, independent on the reader's gain, by referring the backscattered power to a particular state $\Psi_{0}$ of the body process:

$$
p_{S}[\Psi] \equiv \frac{P_{R \leftarrow T}[\Psi]}{P_{R \leftarrow T}\left[\Psi_{0}\right]}=\frac{r c s_{T}\left(\theta^{\prime}, \phi^{\prime}\right)[\Psi]}{r c s_{T}\left(\theta^{\prime}, \phi^{\prime}\right)\left[\Psi_{0}\right]}
$$

Strictly dependent on the environmental condition and on the reader-tag mutual position there is also the turn-on power $P_{i n}^{t o}[\Psi]$, e.g. the minimum input power $P_{i n}$ through the readers antenna forcing the tag to respond. It is directly measurable by the reader and can be derived from (1), considering $P_{R \rightarrow T}=$ $P_{\text {chip }}$ that is the microchip sensitivity.

$$
P_{i n}^{t o}[\Psi]=\left(\frac{\lambda_{0}}{4 \pi d}\right)^{-2} \frac{P_{c h i p}}{G_{R}(\theta, \phi) \eta_{p} G_{T}[\theta, \phi, \Psi(t)] \tau[\Psi(t))}
$$

An alternative processing algorithm for random reader-tag mutual position can be defined by properly combining the forward (1) and the backward (2) powers at turn-on in a way such to drop out the influence of the distance and of the reader and port gains and orientation [5]. A non-dimensional form, denoted as Fingerprint can be hence introduced:

$$
F[\Psi]=\frac{P_{\text {chip }}}{2 \sqrt{P_{R \leftarrow T}[\Psi] P_{\text {in }}^{t o}[\Psi]}}=R_{\text {chip }}\left|Z_{\text {chip }}+Z_{A}(\Psi(t))\right|^{-1}
$$

$F[\Psi]$ is an invariant with respect to distance and to mutual orientation between reader and tag as well as to gains.

\section{MASTERING SENSING}

The sensor-less sensing approach implies that there is no difference from the operative and structural point of view, between antenna and sensor, more precisely the antenna is the sensor and the sensor is the antenna. The sensitivity and selectivity of the system are thus strictly connected to the antenna's feature, in particular to its quality factor, and definitely to its bandwidth. However, due to the high conductivity of body tissues, implanted antennas are typically wideband structures, characterized by high input resistance and low input reactance. Therefore solutions have to be formed to reduce and control its bandwidth.

The design of an implanted radiosensor must be strictly connected to the medical application it will monitor, especially regarding the data inversion curve. Biological processes are characterized by different dynamics in term of time of evolution and amplitude. Some events show very slow and moderate dynamics, requiring a monitoring able to amplify minimal variations in long timescales. In other cases, especially during acute events, the processes are characterized by rapid and large tissues modifications and thus need to be evaluated with sensors able to provide stable responses for a wide range of values. Therefore, the important issue is that in each specific process, or process stage, the inversion curve is able to properly follow the expected dynamics, being for example single-valued, e.g. monotonic, and steep at least in the interesting range of the parameter to be sensed.

The problem raised here, offers several degrees of freedom. In addiction to the tag layout that must be compliant with the anatomical characteristics and able to establish a robust communication link with the external reader, other variables typical of the RFID technology could be considered, such as the microchip properties and the matching conditions.

\section{A. Antenna design and gain}

The design of an antenna suitable for implanted RFID sensor tag is a challenging issue since it should be compliant to both electromagnetic and anatomical needs. First of all, the antenna must be as small and less invasive as possible, biocompatible and able to enhance the interaction of the near field with the tissues of the body. The near field environment within a few centimeters of an antenna in fact strongly influences its electrical characteristics in term of both impedance and gain and thus it is responsible of the sensor activity. By considering that the tissues of the body exhibit significant dielectric loss, primarily due to their water content, converting power from the E-field in to heat, an optimized design configures the antenna to enhance the near E-field over the near $H$-field [6]. However, the increased losses makes more difficult the communication link between the implanted tag and the external reader and consequently an acceptable trade off has to be taken into account during the design procedures.

In addiction to these aspects it necessary to consider also the site of the implants and its anatomy. An important approach, derivable from the military and aerospace world, is that of conformal and structural antennas. In medical context, a conformal antenna could assume for example the shape of a vessel (loop or helix like tag) or be integrated into other implanted devices such as prosthesis, sutures and surgical implants, orthopedic fixing and vascular stents without additional 
radiating element.

\section{B. Microchip choice}

Following [7], it is possible to classify the various commercially available microchips according to their phase angle $Q=X_{\text {chip }} / R_{\text {chip }}$, such as the ratio between the chip input reactance and resistance. Having normalized the power transmission coefficient in (3) by $R_{\text {chip }}^{2}$ one can obtain

$$
\tau=\frac{4 r_{a}}{\mid 1+r_{a}+j Q\left(1+x_{a}\right)}
$$

with $r_{a}=R_{A} / R_{\text {chip }}$ and $x_{a}=X_{A} / X_{\text {chip }}$. After several straightforward mathematical transformations equation (8) assumes the form of an ellipse with the aspect ratio $Q$ and center located at a point $\{(2 / \tau-1),-1\}$.

For a fixed value of power transmission coefficient the higher is the $Q$, the smaller is the ellipses and consequently the narrower is the tag matching bandwidth. Tags matched to microchips with high phase angle show higher sensitivity to the variation of impedance and thus they are more suitable to sensing activity.

\section{Matching condition}

Another interesting approach to manage the sensitivity of an implanted RFID sensor tag comes from the possibility to optimize the matching of the antenna in a specific realization $\Psi_{m}$ of the process $\Psi(t)$ by properly include an antenna adapter. In particular, for a fixed microchip of impedance $Z_{\text {chip }}$, it is possible to impose:

$$
Z_{T}\left(\Psi_{m}\right)=Z_{c h i p}^{*}
$$

which gives $\tau\left(\Psi_{m}\right)=1$. The sensing curves in (5), (6) and (7) are closely related to the matching condition (9) and hence the shape of such curves may be engineered by a proper choice of the parameter $\Psi_{m}$, for instance to emphasize the variation of the early or the late dynamics of the process.

\section{Numerical CASE Study}

In order to check the feasibility of the proposed approach, a realistic clinical case, resembling the liver cancer is here numerically investigated. Liver cancer is a pathological condition characterized by local modifications of the tissues: angiogenesis and disruption of liver vascular architecture produce in comparison to the healthy state a progressive dielectric contrast potentially detectable by an implanted RFID tag. The dielectric contrast in the UHF band could be estimated in about $30 \%$ for the permittivity and about $80 \%$ for the conductivity, as reported in [3].

A stratified FDTD model has been adopted and a $\lambda$-long coated dipole has been implanted into the liver. A mathematical model for an antenna adapter (Fig.2) has been considered in order to match the dipole to different microchips, in particular to the ones with high phase angle in a specific process' state. The implemented model can be viewed as a transformer able to reduce the input resistance and increase the reactance of the dipole, according to the analysis presented in [8], and

\begin{tabular}{c|cc} 
& \multicolumn{2}{|c}{$870 \mathrm{MHz}$} \\
& $\epsilon_{r}$ & $\sigma[\mathrm{S} / \mathrm{m}]$ \\
\hline Skin & 41 & 0.85 \\
Fat & 5.46 & 0.05 \\
Bone & 12.48 & 0.14 \\
Liver & 46.97 & 0.842 \\
Liver Cancer & 60 & 1.5 \\
Internal Organs & 52 & 0.91 \\
Muscle & 55 & 0.93
\end{tabular}

TABLE I

DIELECTRIC PROPERTIES OF HEALTHY AND UNHEALTHY HUMAN TISSUES AT $f=870 M H z$

realizable in practice for example by an inductively coupled loop placed in close proximity of the radiating structure or by adding simple lumped inductor.

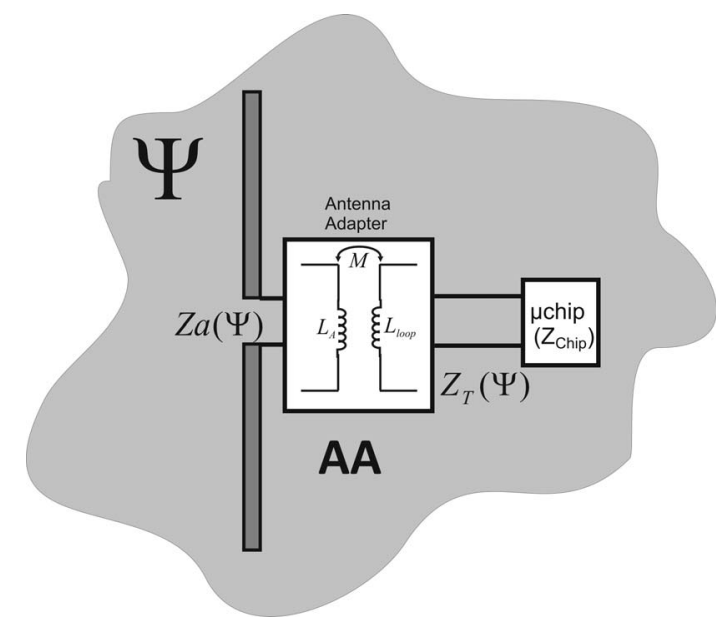

Fig. 2. Reference model: for the implanted tag a fixed radiating structure, such as a dipole has been matched to different microchips by means of an Antenna Adapter. It could be realized for example by adding a inductively coupled loop close to the radiating element or a simple lumped inductor.

Each tissue is characterized by the specific dielectric properties (given in Table I). The cancer has been modeled as a spherical area surrounding the antenna and the whole simulated dynamics is such to change from the absence of malignant tissue to the complete filling of the radiating structure (Fig. 3). Position and size of the tumor has been defined by considering CT data of typical pathologies.

By matching the tag in the intermediate condition $t_{2}$, it is possible to increase the system sensibility to the first part of the process and to detect also small tissues' variation typical of cancer.

Fig. 4 shows the simulated power transmission coefficient in (5) and the fingerprint in (7) referred to the initial condition $t_{1}$ (the time of tag implantation). As previously predicted, the sensing dynamics sensibly increases by using microchip with high phase angle $Q$. For both microchips, the backscattering coefficient is more sensitive to the tumor evolution than fingerprint thanks to its intrinsic dependence on the tag gain $G_{T}[\theta, \phi, \Psi(t)]$ : also for a very low Q microchip the dynamics is about $80 \%$. The variation of gain is a relevant phenomena 


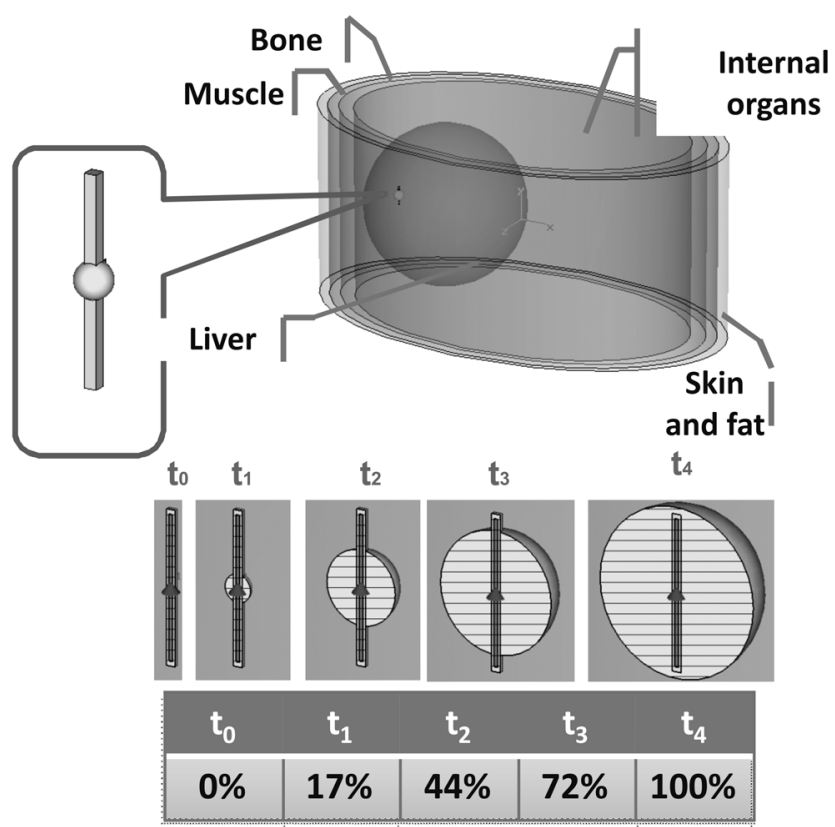

Fig. 3. FDTD model of human liver cancer. The implanted antenna is a $\lambda$-long dipole.

due to the increased conductivity and thus losses of the malignat tissue.
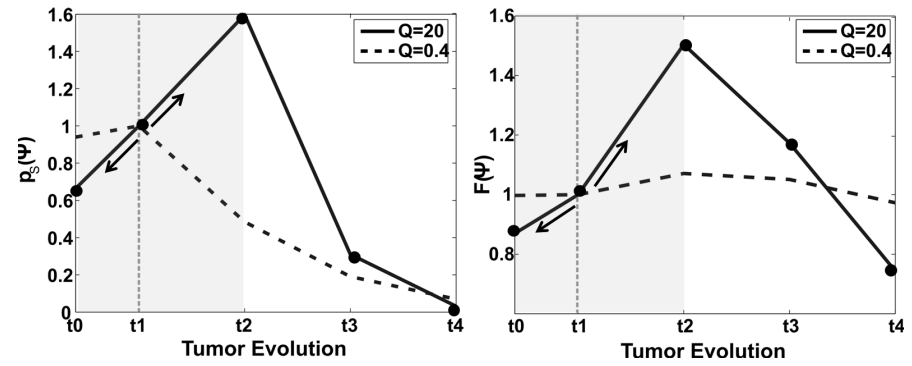

Fig. 4. Backscattered power and Fingerprint of the implanted dipole during the cancer evolution, referred to the reference condition $t_{1}$ with an early stage cancer.

Both inversion curves are monotonic and steep around the reference condition $t_{1}$ allowing the unambiguous monitoring of the process (decrease or increase of the cancer area) in its early stage after the implant. Although the low $Q$ microchip offers a monotonic trend for a wider range of values, it is not suitable for the specific diagnostic purpose since it does not properly amplify the small variations around the reference condition (especially concerning the fingerprint).

The turn-on power $P_{t o}$ referred to a microchip sensitivity $p_{\text {chip }}=32 \mu \mathrm{W}$ ad a distance $d=14 \mathrm{~cm}$ is visible in Fig.5. As expected, the minimum power required to activate the tag is approximately located around the best matching condition $t_{2}$.

\section{EXPERIMENT WITH Body PHANTOM}

The proposed RFID sensing system has been preliminarily experimented by means of an RFID tag implanted into a

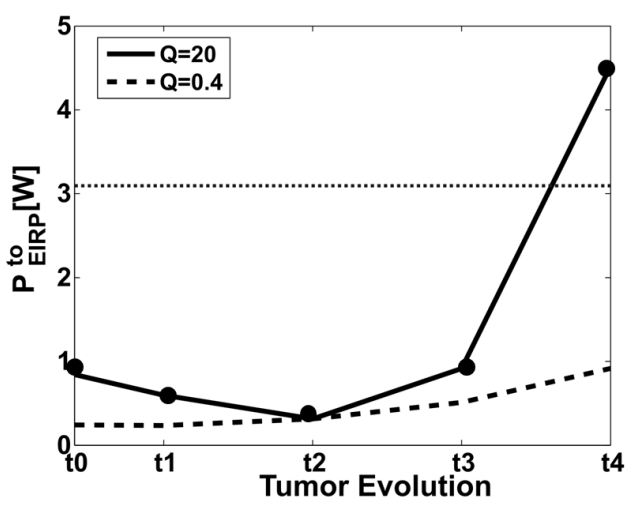

Fig. 5. Turn on power in EIRP of the implanted dipole during the cancer evolution.

human equivalent phantom. A $3 \mathrm{~cm}$ long dipole has been implanted into a liquid phantom (a perspex cylinder with radius $r=5 \mathrm{~cm}$ and high $h=20 \mathrm{~cm}$ ) simulating a generic human district, such as a neck or a limb [9]. The tag is matched to an NXP microchip transponders $(Q=9)$, having $Z_{\text {chip }}=15-j 135 \Omega$ and power sensitivity $P_{\text {chip }}=-15 \mathrm{dBm}$, by means of a $24 n H$ inductor connected in series as in Fig. 6 . The microchip and the matching circuitry are insulated by a teflon tape, in order to avoid short circuit due to the presence of highly conductive liquids.

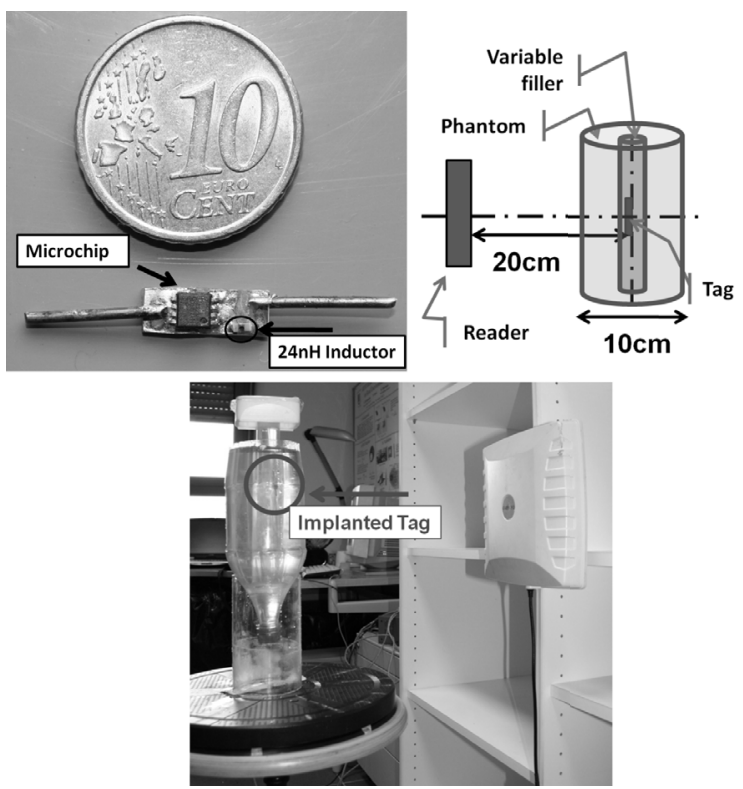

Fig. 6. Implanted dipole and measurement setup

The tag has been placed in the center of a $1 \mathrm{~cm}$ pipe simulating an internal tissue in evolution: the communication and sensing performances of the tag have been evaluated by varying the composition of the liquid inside the pipe, whose properties in term of equivalent complex dielectric permittivity $\bar{\epsilon}=\epsilon_{R e}-j \epsilon_{I m}$ are reported in Tab.II. The tag is best 
matched in condition 4 , such as when it is surrounded only by physiologic solution.

\begin{tabular}{c|c} 
Tissue & Equivalent Complex Permittivity \\
\hline Air & $\bar{\epsilon}_{1}=1$ \\
Alcohol & $\bar{\epsilon}_{2}=27.5-j 10.95$ \\
Phantom & $\bar{\epsilon}_{3}=41.4-j 14.8$ \\
Physiologic Solution $0.157 \mathrm{~N}$ & $\bar{\epsilon}_{4}=75.2-j 35.7$
\end{tabular}

TABLE II

LIQUID PHANTOM PROPERTIES AT 870MHz

All the measurements have been performed by an UHF CAEN reader, connected to a $6-\mathrm{dB}$ gain circular polarized patch antenna.

Fig.7 shows the variation of the turn on-power $P_{t o}$ with the different liquids. The radiation performances of the implanted tag are clearly affected by the tissues composition: considering a reader-tag distance $d=20 \mathrm{~cm}$ the turn-on power linearly ranges from $2.5 \mathrm{~W}$ to $4 \mathrm{~W}$ according to the different liquid fillers.

In the best matching condition and with the maximum power, the implanted tag is readable up to $40 \mathrm{~cm}$.

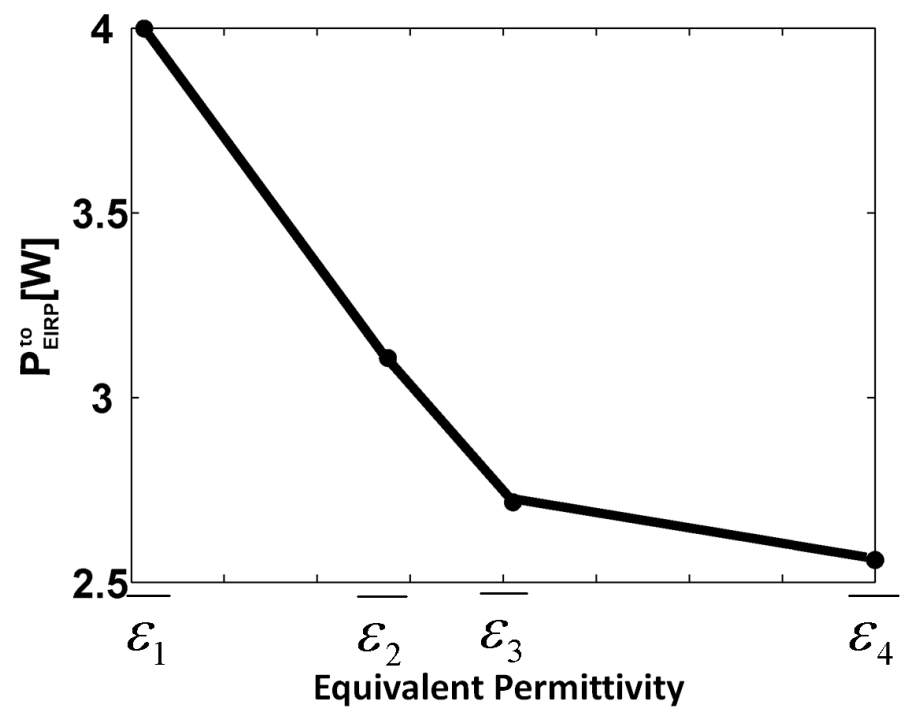

Fig. 7. Turn on power in EIRP of the implanted dipole varying the liquid composition.

\section{CONCLUSION}

Numerical analysis and early experimentations seem to demonstrate that typical biological processes may be sensed by means of implanted passive RFID tags. These phenomena in evolution produce specific and macroscopic effects on the electromagnetic response of the radiosensor, which are detectable by the available technology.

The proposed in-body RFID monitoring could provide various degrees of freedom. Two design approaches have been proposed: the sensitivity of the radiosensor may be enhanced by using high impedance microchips, while the inversion curves may be shaped by properly choosing the impedance matching condition. Those two strategies could be globally controlled and optimized by a RFID Dynamic Design algorithm, where anatomical and physio-pathological considerations are managed together with electromagnetic and system analysis, disclosing new interesting and promising scenarios.

\section{ACKNOWLEDGMENT}

The authors wish to thank prof. Alfredo Marinelli of the Oncology Department of the University of Naples "Federico II" and prof. Lorenzo De Medici of the Radiology Department of San Camillo Hospital, Roma, for inspiration and discussions.

\section{REFERENCES}

[1] G. Marrocco, L. Mattioni, C. Calabrese, "'Multi-port Sensor RFIDs for Wireless Passive Sensing - Basic Theory and Early Simulations"', IEEE Trans. Antennas Propagation.. vol.56, n.8, pp.2691-2702, Aug. 2008

[2] Information available on the website http://www.positiveidcorp.com/.

[3] A. P. ORourke, M. Lazebnik, J. M. Bertram, M.C. Converse, S. C. Hagness, J. G. Webster, D. M. Mahvi, "Dielectric properties of human normal, malignant and cirrhotic liver tissue: in vivo and ex vivo measurements from 0.5 to $20 \mathrm{GHz}$ using a precision open-ended coaxial probe", Phys. Med. Biol. 52 (2007) 47074719

[4] M. Fernndez, D. Semela, J. Bruix, I. Colle, M. Pinzani, J. Bosch, "Angiogenesis in liver disease", Journal of Hepatology Volume 50, Issue 3, Pages 604-620 (March 2009)

[5] G. Marrocco, "Electromagnetic Theory of RFID grids", submitted to IEEE Trans. Antennas Propagation

[6] Y. Rahmat-Samii, J.Kim,Implanted Antennas in Medical Wireless Communications, Morgan \& Claypool Publishers; 1 edition, June 2006.

[7] K. V. S. Rao, P. V. Nikitin, S. F. Lam,'Impedance Matching Concepts in RFID Transponder Design", proceeding of Fourth IEEE Workshop on Automatic Identification Advanced Technologies, pp.39-42, 2005

[8] G.Marrocco, "The Art of UHF RFID Antenna Design: ImpedanceMatching and Size-Reduction Techniques", IEEE Antennas and Propagation Magazine, 2008

[9] G. Hartsgrove,A. Kraszewsky, and A. Surowiec, "'Simulated biological materials for electromagnetic radiation absorption studies", Bioelectromagnetics, vol. 8, no. 4,(1987) pp. 29. 